\title{
A Bayesian Adaptive Design in Cancer Phase I Trials Using Dose Combinations in the Presence of a Baseline Covariate
}

\author{
Márcio Augusto Diniz $(\mathbb{D}$, Sungjin Kim, and Mourad Tighiouart $\mathbb{D}$ \\ Biostatistics and Bioinformatics Research Center, Cedars-Sinai Medical Center 8700 Beverly Blvd, Los Angeles, CA 90048, USA \\ Correspondence should be addressed to Mourad Tighiouart; mourad.tighiouart@cshs.org
}

Received 3 May 2018; Revised 27 July 2018; Accepted 29 August 2018; Published 1 November 2018

Academic Editor: Ash Abebe

Copyright (C) 2018 Márcio Augusto Diniz et al. This is an open access article distributed under the Creative Commons Attribution License, which permits unrestricted use, distribution, and reproduction in any medium, provided the original work is properly cited.

\begin{abstract}
A Bayesian adaptive design for dose finding of a combination of two drugs in cancer phase I clinical trials that takes into account patients heterogeneity thought to be related to treatment susceptibility is described. The estimation of the maximum tolerated dose (MTD) curve is a function of a baseline covariate using two cytotoxic agents. A logistic model is used to describe the relationship between the doses, baseline covariate, and the probability of dose limiting toxicity (DLT). Trial design proceeds by treating cohorts of two patients simultaneously using escalation with overdose control (EWOC), where at each stage of the trial, the next dose combination corresponds to the $\alpha$ quantile of the current posterior distribution of the MTD of one of two agents at the current dose of the other agent and the next patient's baseline covariate value. The MTD curves are estimated as function of Bayes estimates of the model parameters at the end of trial. Average DLT, pointwise average bias, and percent of dose recommendation at dose combination neighborhoods around the true MTD are compared between the design that uses the covariate and the one that ignores the baseline characteristic. We also examine the performance of the approach under model misspecifications for the true dose-toxicity relationship. The methodology is further illustrated in the case of a prespecified discrete set of dose combinations.
\end{abstract}

\section{Introduction}

Despite the promise observed in preclinical experiments and initial high response rates, a large number of targeted drugs have not been successful in providing reproducible improvements in survival in patients with cancer when used as single agents. [1] In addition, targeted therapies do not work for every patient since they rely on the presence of the target. Therefore, chemotherapy and radiotherapy approaches are still the backbone of cancer treatment for tumors after surgical excision. These conventional cancer therapies may be combined with targeted agents to enhance treatment efficacy.

Statistical methodologies for designing phase I clinical trials for drug combinations have been studied extensively in the past decade [2-13]. These methods assume that the patient population is homogeneous of treatment tolerance and every patient should be treated at a dose combination corresponding to a predefined target probability of DLT (dose limiting toxicity). Therefore, an additional layer of complexity in specifying the dose-toxicity relationship given a baseline covariate is needed for drug combinations.

Strategies of drug allocation that accommodate individual patient needs have been used in [14-18] for single agent trials. Statistical designs allowing individualized maximum tolerable dose (MTD) determination in single agent cancer phase I trials have also been proposed and implemented in real trials by a number of authors for two groups with no prior knowledge of ordering $[19,20]$, for two prior ordered groups $[21,22]$ and two or more prior partially ordered groups [23, 24]. In general, ignoring the heterogeneity between groups can lead to higher toxicities in the most severely impaired group, statistical bias, and inefficiency of the MTD estimate for both groups.

In this work, we extend the design described by Tighiouart et al. [25] using escalation with overdose control (EWOC) principle [26], by treating cohorts of two patients simultaneously and accounting for patient baseline binary covariate. We assume that we do not have prior knowledge of the ordering between groups, but they will be ordered in 
the sense that the probability of toxicity for one group is always a constant shift from the probability of toxicity for the second group at the same dose. In this way, patients with different covariate values will have parallel MTD curves. This assumption is mathematically convenient and allows us to use parsimonious models due to the small sample size constraints in cancer phase I trials.

This paper is organized as follows. Section 2 will describe the dose-toxicity model and trial design for continuous dose levels. In Section 3, we evaluate the performance of the proposed method by assessing the safety of the trial design and the efficiency of the estimate of the MTD curve. The methodology is extended for discrete dose combinations in Section 4. Discussions will be presented in Section 5.

\section{Model}

2.1. Dose-Toxicity Model. We propose a parametric model to identify tolerable dose combinations of two synergistic drugs $A$ and $B[10-12,25,27]$ given a patient with a binary baseline covariate value of $z$ :

$$
\operatorname{Prob}(\delta=1 \mid x, y, z)=F(\mu+\beta x+\gamma y+\lambda z+\eta x y)
$$

where $\delta$ is the indicator of DLT, $(x, y)$ are the continuous dose levels of agents $A$ and $B$, respectively, assuming values in $\left[X_{\text {min }}, X_{\text {max }}\right] \times\left[Y_{\text {min }}, Y_{\text {max }}\right], z$ is a binary baseline covariate value, and $F$ is a known cumulative distribution function.

We assume partial ordering of the probability of DLT, i.e., it is a nondecreasing function of the dose of any one of the agents when the other one is held constant for $z=0,1$ and we also assume synergism between the two drugs. These assumptions are translated into constrains in the parameter space given by $\beta, \gamma>0$, and $\eta \geq 0$, respectively. The MTD $C_{z}$ for a patient with covariate value $\mathrm{z}$ is defined as the set of combinations $\left(x^{*}, y^{*}\right)$ such that

$$
\operatorname{Prob}\left(\delta=1 \mid x^{*}, y^{*}, z\right)=\theta \text {. }
$$

The target probability of DLT, $\theta$, is set relatively high when the DLT is a reversible or nonfatal condition, and low when it is life threatening. Using (1) and (2), the $\operatorname{MTD} C_{z}$ is

$$
\begin{aligned}
C_{z} & =\left\{\left(x^{*}, y^{*}\right) \in[0,1]^{2}: y^{*}\right. \\
& \left.=\frac{F^{-1}(\theta)-\mu-\beta x^{*}-\lambda z}{\gamma+\eta x^{*}}\right\} .
\end{aligned}
$$

We reparametrize model (1) to allow a more meaningful prior elicitation. Assuming that $\left[X_{\min }, X_{\max }\right] \times\left[Y_{\min }, Y_{\max }\right]$ will be standardized to be in $[0,1] \times[0,1], \rho_{000}$, the probability of DLT at the minimum available doses of agents $A$ and $B$ for a patient with covariate value $z=0 ; \rho_{100}$, the probability of DLT when the level of $\operatorname{drug} A$ is $X_{\text {max }}$, the level of $\operatorname{drug} B$ is $Y_{\min }$ and $z=0 ; \rho_{101}$, the probability of DLT when the level of $\operatorname{drug} A$ is $X_{\text {max }}$, the level of $\operatorname{drug} B$ is $Y_{\text {min }}$ and $z=1 ; \rho_{010}$, the probability of DLT when the level of $\operatorname{drug} A$ is $X_{\text {min }}$, the level of drug $B$ is $Y_{\text {max }}$ and $z=0$; and the interaction parameter $\eta$. It follows that

$$
\begin{aligned}
& \mu=F^{-1}\left(\rho_{000}\right) \\
& \beta=F^{-1}\left(\rho_{100}\right)-F^{-1}\left(\rho_{000}\right) \\
& \gamma=F^{-1}\left(\rho_{010}\right)-F^{-1}\left(\rho_{000}\right) \\
& \lambda=F^{-1}\left(\rho_{101}\right)-F^{-1}\left(\rho_{100}\right) .
\end{aligned}
$$

Notice that $\beta, \gamma>0$ implies that $\rho_{000}<\min \left(\rho_{100}, \rho_{010}\right)$. The MTD set given in (3) can be presented as

$$
\begin{aligned}
C_{z} & =\left\{\left(x^{*}, y^{*}\right) \in[0,1]^{2}: y^{*}\right. \\
& \left.=\frac{G\left(\theta, \rho_{000}\right)-\left(G\left(\rho_{100}, \rho_{000}\right)\right) x^{*}-\left(G\left(\rho_{101}, \rho_{100}\right)\right) z}{G\left(\rho_{010}, \rho_{000}\right)+\eta x^{*}}\right\},
\end{aligned}
$$

where $G(a, b)=F^{-1}(a)-F^{-1}(b)$.

Let $D_{n}=\left\{\left(x_{i}, y_{i}, z_{i}, \delta_{i}\right), i=1, \ldots, n\right\}$ be the data after enrolling $n$ patients in the trial. The likelihood function under the reparametrization is

$$
\begin{aligned}
& L\left(\rho_{000}, \rho_{100}, \rho_{101}, \rho_{010}, \eta \mid D_{n}\right) \\
& =\prod_{i=1}^{n}\left(H\left(\rho_{000}, \rho_{100}, \rho_{101}, \rho_{010}, \eta ; x_{i}, y_{i}, z_{i}\right)\right)^{\delta_{i}} \\
& \quad \times\left(1-H\left(\rho_{000}, \rho_{100}, \rho_{101}, \rho_{010}, \eta ; x_{i}, y_{i}, z_{i}\right)\right)^{1-\delta_{i}}
\end{aligned}
$$

where

$$
\begin{aligned}
H & \left(\rho_{000}, \rho_{100}, \rho_{101}, \rho_{010}, \eta ; x_{i}, y_{i}, z_{i}\right)=F\left(F^{-1}\left(\rho_{000}\right)\right. \\
& +\left(F^{-1}\left(\rho_{100}\right)-F^{-1}\left(\rho_{000}\right)\right) x_{i} \\
& +\left(F^{-1}\left(\rho_{010}\right)-F^{-1}\left(\rho_{000}\right)\right) y_{i} \\
& \left.+\left(F^{-1}\left(\rho_{101}\right)-F^{-1}\left(\rho_{100}\right)\right) z_{i}+\eta x_{i} y_{i}\right)
\end{aligned}
$$

2.2. Prior and Posterior Distributions. We consider the priors $\rho_{100} \sim \operatorname{Beta}\left(a_{1}, b_{1}\right), \rho_{010} \sim \operatorname{Beta}\left(a_{3}, b_{3}\right), \rho_{101} \sim$ $\operatorname{Bet} a\left(a_{2}, b_{2}\right)$, and conditional on $\rho_{100}, \rho_{010}, \rho_{000} / \min \left(\rho_{100}\right.$, $\left.\rho_{010}\right) \sim \operatorname{Beta}\left(a_{0}, b_{0}\right)$, and $\eta \sim \operatorname{Gamma}(a, b)$ with mean $E(\eta)=$ $a / b$ and variance $\operatorname{var}(\eta)=a / b^{2}$. As described in [25], vague Beta priors are achieved by taking $a_{j}=b_{j}=1, j=0,1,2,3$ while a vague Gamma prior is chosen with mean of 21 and variance of 540 . The posterior distribution is given by,

$$
\begin{aligned}
& \pi\left(\rho_{000}, \rho_{100}, \rho_{101}, \rho_{010}, \eta \mid D_{n}\right) \\
& \propto \prod_{i=1}^{n}\left(H\left(\rho_{000}, \rho_{100}, \rho_{101}, \rho_{010}, \eta ; x_{i}, y_{i}, z_{i}\right)\right)^{\delta_{i}} \\
& \quad \times\left(1-H\left(\rho_{000}, \rho_{100}, \rho_{101}, \rho_{010}, \eta ; x_{i}, y_{i}, z_{i}\right)\right)^{1-\delta_{i}} \\
& \quad \times \pi\left(\rho_{000} \mid \rho_{100}, \rho_{010}\right) \pi\left(\rho_{100}\right) \pi\left(\rho_{101}\right) \pi\left(\rho_{010}\right) \pi(\eta) .
\end{aligned}
$$

We used JAGS [28] to sample from the posterior distribution. 
2.3. Trial Design. The algorithm for dose escalation/deescalation is similar to one discussed in $[11,25]$ with the additional binary covariate information. It uses the EWOC principle [26] where at each stage of the trial, we seek a dose of one agent using the current posterior distribution of the MTD of the agent given the current dose of the other agent and the next patient's baseline covariate value. For instance, if agent $A$ is held constant at level $x$, the dose of agent $B$ is $y$ such that the posterior probability that $y$ exceeds the MTD of agent $B$ given the dose of agent $A=x$ and covariate value $Z=z$ is bounded by a feasibility bound $\alpha$. Cohorts of two patients are enrolled simultaneously receiving different dose combinations. Specifically, the design proceeds as follows.

(1) Let $D_{2}=\left\{\left(x_{1}, y_{1}, z_{1}, \delta_{1}\right),\left(x_{2}, y_{2}, z_{2}, \delta_{2}\right)\right\}$ be the data from the first cohort of two patients such that each patient receives the same dose combination $\left(x_{i}, y_{i}\right)=$ $\left(X_{\min , A}, Y_{\min , B}\right)=(0,0)$ for $i=1,2$.

(2) In the second cohort of two patients, patient 3 receives dose $\left(x_{1}, y_{3}\right)$ and patient 4 receives dose $\left(x_{4}, y_{2}\right)$. If $z_{3}=z_{1}$ or $z_{3}=z_{2}, y_{3}$ is the $\alpha$ th percentile of $\pi\left(\Gamma_{B \mid A=x_{1}, Z=z_{3}} \mid D_{2}\right)$. Otherwise, patient 3 receives the minimum dose combination $\left(X_{\min , A}, Y_{\min , B}\right)=(0,0)$. If $z_{4}=z_{1}$ or $z_{4}=z_{2}, x_{4}$ is the $\alpha$ th percentile of $\pi\left(\Gamma_{A \mid B=y_{2}, Z=z_{4}} \mid D_{2}\right)$. Otherwise, patient 4 receives the minimum dose combination $\left(X_{\min , A}, Y_{\min , B}\right)=$ $(0,0)$. In general, the first time a patient is assigned to a given group defined by the binary covariate $z$ always receives the minimum dose combination $\left(X_{\min , A}, Y_{\min , B}\right)$ no matter how many patients have been treated in the other group, as described in [20]. Here, $\pi\left(\Gamma_{B \mid A=x_{1}, Z=z_{3}} \mid D_{2}\right)$ is the posterior distribution of the MTD of agent $B$ given that the level of agent $A$ is $x_{1}$ and the baseline covariate value of patient 3 is $z_{3}$, given the data $D_{2} \cdot \pi\left(\Gamma_{A \mid B=y_{2}, Z=z_{4}} \mid D_{2}\right)$ is defined similarly. $\Gamma_{B \mid A=x}$ and $\Gamma_{A \mid B=y}$ can be expressed in terms of $\rho_{000}, \rho_{100}, \rho_{101}$, and $\rho_{010}$.

(3) In the $i$-th cohort of two patients,

(a) If $i$ is even, patient $(2 i-1)$ receives dose $\left(x_{2 i-3}, y_{2 i-1}\right)$ and patient $2 i$ receives dose

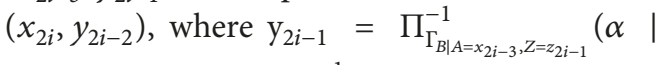
$\left.D_{2 i-2}\right)$ and $x_{2 i}=\Pi_{\Gamma_{A \mid B=y_{2 i-2}, Z=z_{2 i}}^{-1}}\left(\alpha \mid D_{2 i-2}\right)$. Here, $\Pi_{\Gamma_{A \mid B=y, Z=z}}^{-1}(\alpha \quad D)$ is the inverse cumulative distribution function of the posterior distribution, $\pi\left(\Gamma_{A \mid B=y, Z=z} \mid D\right)$.

(b) Similarly, if $i$ is odd, patient $(2 i-1)$ receives dose $\left(x_{2 i-1}, y_{2 i-3}\right)$ and patient $2 i$ receives dose $\left(x_{2 i-2}, y_{2 i}\right)$, where $x_{2 i-1}=\prod_{\Gamma_{A \mid B=y_{2 i-3}, Z=z_{2 i-1}}^{-1}}(\alpha \mid$

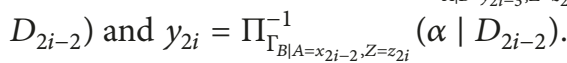

(4) Repeat step (3), until $n$ patients are enrolled in the trial subject to the following stopping rule.

If the $\alpha$ th percentile of $\pi\left(\Gamma_{A \mid B=y, Z=z} \mid D\right)$ or $\pi\left(\Gamma_{B \mid A=x, Z=z} \mid D\right)$ is less than 0 or greater than 1 , the recommended dose for the next patient is 0 or 1 , respectively. In steps (2) and (3) above, a dose escalation is further restricted to be no more than a prespecified fraction of the dose range of the corresponding agent.

Stopping Rule. It is sufficient to evaluate a stopping rule for safety at the minimum dose combination because of the partial ordering assumption. The probability of DLT of all doses for both agents will be higher than $\theta$ if the probability at the minimum dose is higher than $\theta$.We stop enrollment to the trial if $P\left(P(D L T \mid(x, y)=(0,0), z) \geq \theta+\delta_{1} \mid\right.$ data $)>\delta_{2}$, i.e., if the posterior probability that the probability of DLT at the minimum available dose combination in the trial exceeds the target probability of DLT is high for $z=0,1$. The design parameters $\delta_{1}$ and $\delta_{2}$ are chosen to achieve desirable model operating characteristics. At the completion of the trial, an estimate of the MTD curve for $z=0,1$ is obtained using (5) as

$$
\begin{aligned}
\widehat{C}_{z} & =\left\{\left(x^{*}, y^{*}\right) \in[0,1]^{2}: y^{*}\right. \\
& \left.=\frac{G\left(\theta, \widehat{\rho}_{000}\right)-\left(G\left(\widehat{\rho}_{100}, \hat{\rho}_{000}\right)\right) x^{*}-\left(G\left(\widehat{\rho}_{101}, \widehat{\rho}_{100}\right)\right) z}{G\left(\widehat{\rho}_{010}, \widehat{\rho}_{000}\right)+\widehat{\beta}_{4} x^{*}}\right\} .
\end{aligned}
$$

where $G(a, b)=F^{-1}(a)-F^{-1}(b), \hat{\rho}_{000}, \hat{\rho}_{100}, \hat{\rho}_{101}, \hat{\rho}_{010}$, and $\hat{\eta}$ are the posterior medians given the data $D_{n}$.

\section{Simulation Studies}

3.1. Simulation Set-Up and Scenarios. We present four scenarios for the true MTD curves as shown in Figure 1. The first scenario (a) is a case where the two true MTD curves for two groups are parallel and close to the minimum doses with $\rho_{010}$ and $\rho_{100}$ equal to each other and slightly higher than $\theta$; the second scenario (b) is a case where the two true MTD curves for two groups are parallel but very close to each other; the third scenario (c) is a case where two true MTD curves for two groups are not parallel, and the last scenario (d) is a case where the two true MTD curves are parallel but lie far apart from each other and close to the maximum doses with $\rho_{010}$ and $\rho_{100}$ equal and largely lower than $\theta$.

In addition, toxicity responses are generated assuming four link functions allowing us to evaluate misspecification: (i) logistic, $F(u)=\left(1+e^{-u}\right)^{-1}$, (ii) probit, $F(\mathrm{u})=\Phi(u)$, where $\Phi(\cdot)$ is the c.d.f. of the standard normal distribution, (iii) normal, $F(u)=\Phi(u / \sigma)$ with $\sigma=2$, and (iv) complementary $\log -\log , F(u)=1-e^{-e^{u}}$, where the parameter values of $\mu, \beta$, $\gamma, \lambda$, and $\eta$ were selected in such a way that they all have the same true MTD curve.

For each scenario, 1000 trials were simulated with the logistic link function as the working model, the target probability of DLT is fixed at $\theta=0.33$, the trial sample size is $n=40$ patients with 20 patients in each group, $\delta_{1}=0.05$ and $\delta_{2}=$ 0.8 . Vague priors for the parameters $\left(\rho_{000}, \rho_{100}, \rho_{101}, \rho_{010}, \eta\right)$ were chosen. A variable feasibility bound $\alpha$ was started from 0.25 and increased by 0.05 each time when we compute the dose for the next patient until $\alpha$ was reached 0.5 [29]. A dose escalation is restricted to be no more than $20 \%$ of the dose range of the corresponding agent. 
3.2. Design Operating Characteristics. In order to assess the performance of this method when designing a prospective trial, we evaluate its operating characteristics by comparing the following three designs.

(i) Design using a covariate (WC): patients are accrued to the trial sequentially and the dose combinations given to the next cohort of patients are calculated assuming model (1).

(ii) Design ignoring the covariate (IC): patients are accrued to the trial sequentially and the dose combinations given to the next cohort of patients are calculated assuming model (1) without the covariate, i.e., as in [25].

(iii) Design using parallel trials (PT): in each group, patients are accrued to the trial sequentially and model (1) without the covariate is implemented in each group.

We assume that we have balanced groups given a fixed sample size in which it is possible to carry out two separate studies.

3.2.1. Safety and Efficiency. We evaluate operating characteristics introduced by Tighiouart et al. (2014, 2017) [11, 25]. Safety is assessed through the average percent of DLTs across all trials and the percent of trials that have a DLT rate exceeding $\theta+0.1$.

Efficiency is assessed using an overall MTD estimate, pointwise average bias, and percentage of selection. The overall MTD estimate is based on all trials:

$$
\bar{C}_{z}=\left\{\left(x^{*}, y^{*}\right) \in[0,1]^{2}: y^{*}=\frac{F^{-1}(\theta)-F^{-1}\left(\bar{\rho}_{000}\right)-\left(F^{-1}\left(\bar{\rho}_{100}\right)-F^{-1}\left(\bar{\rho}_{000}\right)\right) x^{*}-\left(F^{-1}\left(\bar{\rho}_{101}\right)-F^{-1}\left(\bar{\rho}_{100}\right)\right) z}{F^{-1}\left(\bar{\rho}_{010}\right)-F^{-1}\left(\bar{\rho}_{000}\right)+\bar{\eta} x^{*}}\right\} .
$$

where $z=0,1, F(\cdot)$ is the logistic function and $\bar{\rho}_{000}, \bar{\rho}_{100}, \bar{\rho}_{101}, \bar{\rho}_{010}$, and $\bar{\eta}$ are the average posterior medians of the parameters $\rho_{000}, \rho_{100}, \rho_{101}, \rho_{010}$, and $\eta$ from all 1000 trials, respectively.

The pointwise average relative minimum distance from the true MTD curve $C_{z, \text { true }}$ to the estimated MTD curve for $z=0,1$ is defined as

$$
d_{z(x, y)}=m^{-1} \sum_{i=1}^{m} d_{z(x, y)}^{(i)}
$$

wherein

$$
\begin{aligned}
d_{z(x, y)}^{(i)}= & \operatorname{sign}\left(y^{\prime}-y\right) \\
& \times \min _{\left\{\left(x^{*}, y^{*}\right):\left(x^{*}, y^{*}\right) \in C_{z, i}\right\}} \sqrt{\left(x-x^{*}\right)^{2}+\left(y-y^{*}\right)^{2}}
\end{aligned}
$$

for every point $(x, y) \in C_{z, \text { true }}, y^{\prime}$ is such that $\left(x, y^{\prime}\right) \in C_{z, i}$ for all $(x, y) \in C_{z, \text { true }}$, and $C_{z, i}$ is the estimated MTD curve with binary covariate $z$ for trial $i$.

The percentage of selection for $z=0,1$ uses the differences defined in (12):

$$
P_{z(x, y)}=m^{-1} \sum_{i=1}^{m} I\left(\left|d_{z(x, y)}^{(i)}\right| \leq p \Delta_{z}(x, y)\right)
$$

where $\Delta_{z}(x, y)$ is the Euclidean distance between the minimum dose combination $(0,0)$ and the point $(x, y)$ on the true MTD curve for $z=0,1$ and $0<p<1$.

\subsection{Results}

3.3.1. Trial Safety. Table 1 shows that the overall average percent of DLTs is always less than $\theta=0.33$ varying between $16.84 \%$ and $30.42 \%$ for the WC design, and $21.55 \%$ and $30.10 \%$ for the IC design across four scenarios. In the group with $Z=0$, the average percent of DLTs varies between $5.63 \%$ and $21.68 \%$ for WC design, $2.89 \%$ and $19.07 \%$ for IC design, and $6.43 \%$ and $21.95 \%$ for PT design. Safety becomes a concern when $Z=1$ for IC design because high values of average percent of DLT are observed (varying between $32.37 \%$ and $46.15 \%)$. On the other hand, the average percent of DLT for all scenarios goes between $28.04 \%$ and $39.17 \%$ for WC design, and $14.54 \%$ and $29.55 \%$ for PT design. These rates are similar when using the true and misspecified models. In addition, the highest value of the percent of trials with an excessive rate of DLT as defined by a DLT rate exceeding $\theta+0.1$ is $0.1 \%$ for WC design, $0.4 \%$ for IC design, and $0.0 \%$ for PT design in the group with $Z=0$ while this value is higher for $Z=1$ is $31.5 \%$ for WC design, $65.8 \%$ for IC design, and $1.8 \%$ for PT design. Thus, we conclude that the methodology is safe for WC and PT designs, but not for IC design. The other three misspecified models are shown in Table S1.

3.3.2. Trial Efficiency. Figure 1 shows the true and estimated MTD curves for each group of patients under the four scenarios (a)-(d) when using the three studied designs. The estimated MTD curves were obtained using (10) and DLT responses were simulated using the logistic link function. The estimated MTD curves are fairly close to the true MTD curves when accounting for a significant baseline covariate (scenarios a, c, d) using the WC and PT designs. When ignoring the covariate, the estimated MTD curve tends to be in between the true MTD curves. This shows that when 

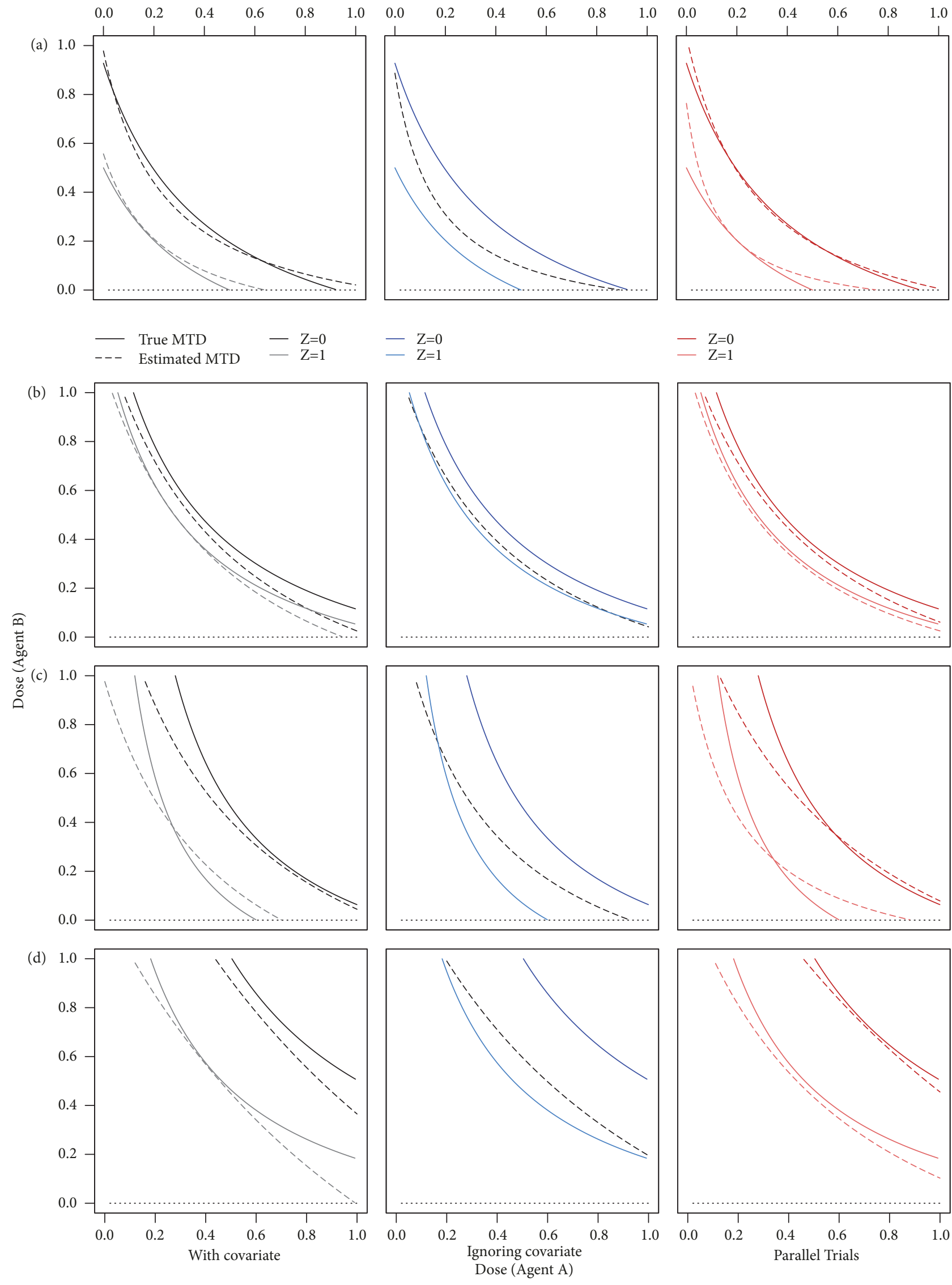

d)

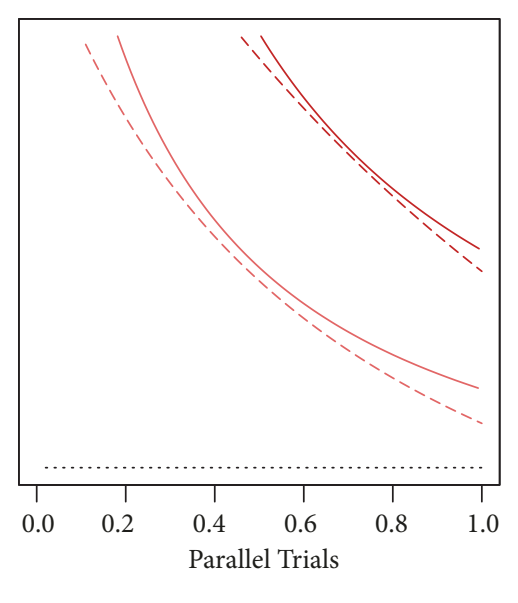

FIgURE 1: True and estimated MTD curves from $m=1000$ simulated trials with designs using a covariate (WC), ignoring the covariate (IC), and parallel trials (PT) under four scenarios (a)-(d). 
TABLE 1: Operating characteristics summarizing trial safety for designs using a covariate (WC), ignoring the covariate (IC), and parallel trials (PT) considering continuous dose combinations, $\theta=0.33$.

\begin{tabular}{|c|c|c|c|c|}
\hline \multirow[t]{2}{*}{ Scenario } & \multirow[t]{2}{*}{ Design } & \multicolumn{3}{|c|}{$\begin{array}{c}\text { Average } \% \text { DLTs } \\
(\% \text { Trials: DLT rate }<\theta-0.1 ; \% \text { Trials: DLT rate }>\theta+0.1)\end{array}$} \\
\hline & & Overall & $Z=0$ & $Z=1$ \\
\hline \multirow{3}{*}{ (a) } & WC & $30.42(7.1 ; 0.5)$ & $21.68(56.6 ; 0.0)$ & $39.17(0.4 ; 31.5)$ \\
\hline & IC & $30.10(8.6 ; 0.4)$ & $14.05(86.7 ; 0.1)$ & $46.15(0.0 ; 65.8)$ \\
\hline & PT & - & $21.95(56.6 ; 0.0)$ & $29.55(10.9 ; 1.8)$ \\
\hline \multirow{3}{*}{ (b) } & WC & $24.34(45.4 ; 0.0)$ & $19.47(73.8 ; 0.1)$ & $29.21(13.4 ; 1.8)$ \\
\hline & IC & $25.72(34.6 ; 0.0)$ & $19.07(68.1 ; 0.4)$ & $32.37(10.2 ; 11.7)$ \\
\hline & PT & - & $16.87(88.3 ; 0.0)$ & $19.04(78.0 ; 0.0)$ \\
\hline \multirow{3}{*}{ (c) } & WC & $24.15(47.7 ; 0.0)$ & $13.98(93.6 ; 0.0)$ & $34.32(2.7 ; 11.0)$ \\
\hline & IC & $24.90(41.6 ; 0.0)$ & $8.60(97.3 ; 0.0)$ & $41.21(0.8 ; 42.6)$ \\
\hline & PT & - & $14.78(96.5 ; 0.0)$ & $22.21(55.2 ; 0.1)$ \\
\hline \multirow{3}{*}{ (d) } & WC & $16.84(98.0 ; 0.0)$ & $5.63(100.0 ; 0.0)$ & $28.04(15.4 ; 0.0)$ \\
\hline & IC & $21.55(73.3 ; 0.0)$ & $2.89(100.0 ; 0.0)$ & $40.22(1.0 ; 36.8)$ \\
\hline & PT & - & $6.43(100.0 ; 0.0)$ & $14.54(97.6 ; 0.0)$ \\
\hline
\end{tabular}

Scenario $\left(\rho_{000}, \rho_{100}, \rho_{101}, \rho_{010}, \eta\right)$ : (a) $(0.01,0.4,0.8,0.4,10)$, (b) $(0.005,0.1,0.2,0.1,10),\left(\right.$ c) $(0.005,0.2,0.7,0.01,10)$, and $(\mathrm{d})\left(10^{-4}, 10^{-3}, 0.05,10^{-3}, 10\right)$.

the two MTD curves are well separated, not accounting for a baseline covariate results in suboptimal MTD curve estimation for the group of patients with high tolerance and a too toxic MTD curve recommendation for the other group.

Figure 2 displays the pointwise average relative minimum distance from the true MTD curve to the estimated MTD curve as defined by (11) under the four scenarios (a)-(d) when the DLT responses are simulated from the true and the other three misspecified models, respectively. This is a measure of pointwise bias for the MTD estimate. In the first scenario (a), the maximum absolute pointwise bias is 0.101 for $Z=0$ and 0.099 for $Z=1$. For WC design, the pointwise bias is negligible for low dose combinations and increases as we move away from the minimum dose combination with higher values when $Z=0$ then $Z=1$. For PT design, the pointwise bias is almost constant when $Z=0$, such that it is lower than for WC design at the edges of the MTD curve and presents $\mathrm{U}$-shape when $Z=1$ with higher values than for WC design at the minimum dose combination and the edges of the MTD curve. In scenario (b), the maximum absolute pointwise bias is 0.069 for $Z=0$ and 0.066 for $Z=1$. WC and PT designs show $U$-shape pointwise bias with higher values for WC design than for PT design as we increase the dose combinations in any direction when $Z=0,1$. In scenario (c), the maximum absolute pointwise bias is 0.181 for $Z=0$ and 0.155 for $Z=1$. For WC design, the pointwise bias is negative for low dose combinations and approximates to zero as we increase the dose combination in any direction; For PT design, the bias is negative for low dose combinations and becomes positive until reaching the same initial magnitude when $Z=1$ and a plateau lower than the initial magnitude when $Z=0$ as we increase dose combinations in any direction. In scenario (d), the maximum absolute pointwise bias is 0.21 for $Z=0$ and 0.139 for $Z=1$. WC and PT designs are similar to each other, with WC showing higher pointwise bias for doses at the edge of MTD curve when $Z=1$. IC design presents higher pointwise bias than for WC and PT designs in all scenarios. The other three misspecified models are shown in Figure S1.

Figure 3 shows the pointwise percent of trials for which the minimum distance from the true MTD curve to the estimated MTD curve is no more than $(100 \times p) \%$ of the true MTD for $p=0.2$ as defined by (13). This can be interpreted as the percent of MTD recommendation for a given tolerance $p$. Under the first scenario (a), the percent of trials with correct MTD recommendation within 20\% of the true value of the MTD varies between $62.6 \%$ and $99.9 \%$ using WC and PT designs, while it varies more widely between $28.6 \%$ and $100 \%$ for the IC design when the toxicities are generated from the true and misspecified models. The WC and PT design presents similar results to each other, with WC design showing slightly lower values than for PT design at the minimum dose combination. Under the second scenario (b), the percent of recommendation is similar between all designs varying between $84.9 \%$ and $97.6 \%$ for the WC design, $89.8 \%$ and $99.3 \%$ for the IC design, and $79 \%$ and $100 \%$ for the PT design. The WC design presents somewhat lower values than for IC design when $Z=1$ at the minimum dose combination and at central part of the MTD curve when $Z=0$. Under the scenario (c), the percent of recommendation is between $69.0 \%$ and $95.1 \%$ for the WC design, $67.1 \%$ and $95.3 \%$ for the PT design, while it is between $15.6 \%$ and $98.5 \%$ for IC design. The IC design is notably worse than WC and PT designs, except at the minimum dose combination when $Z=1$; The percent of recommendation is always lower for PT design than for WC design when $Z=0$ and at the edges of the MTD curve when $Z=1$. In scenario (d), the percent of recommendation varies between $68.7 \%$ and $95.5 \%$ for WC design, $88.2 \%$ and $98.0 \%$ for the PT design, $50.7 \%$ and $89.2 \%$ for the IC design. As it was observed in the other scenarios, IC design performs worse than $\mathrm{WC}$ and PT designs. The PT design presents higher values than for WC design at the minimum dose combination when $Z=0$ and at the edges of 
(a)

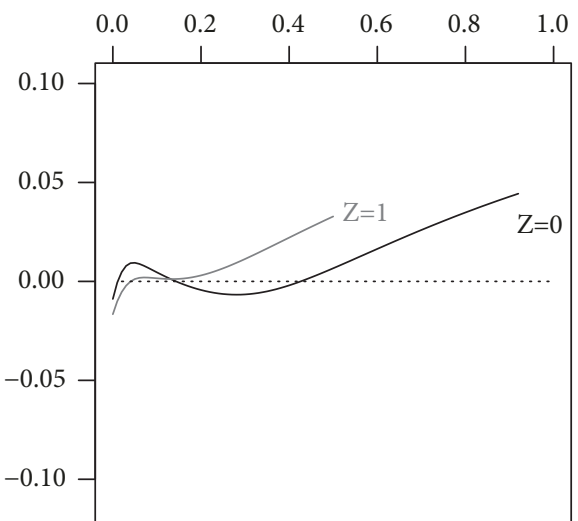

(b)

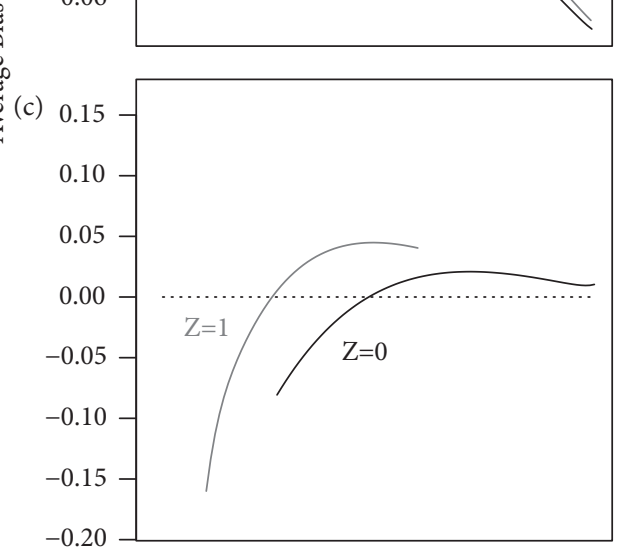

(d)

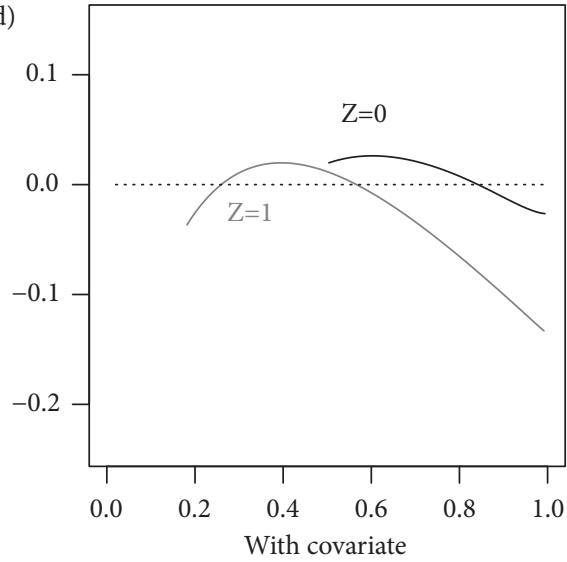

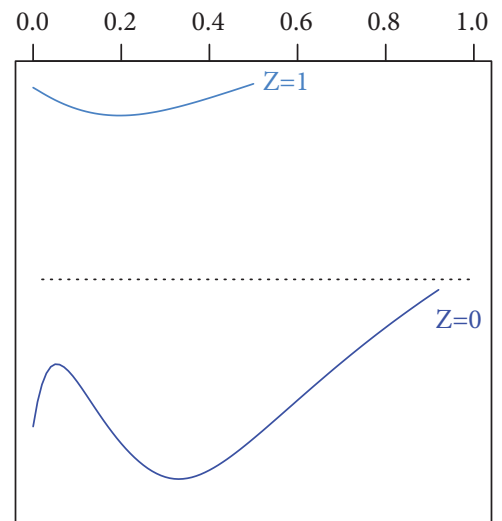
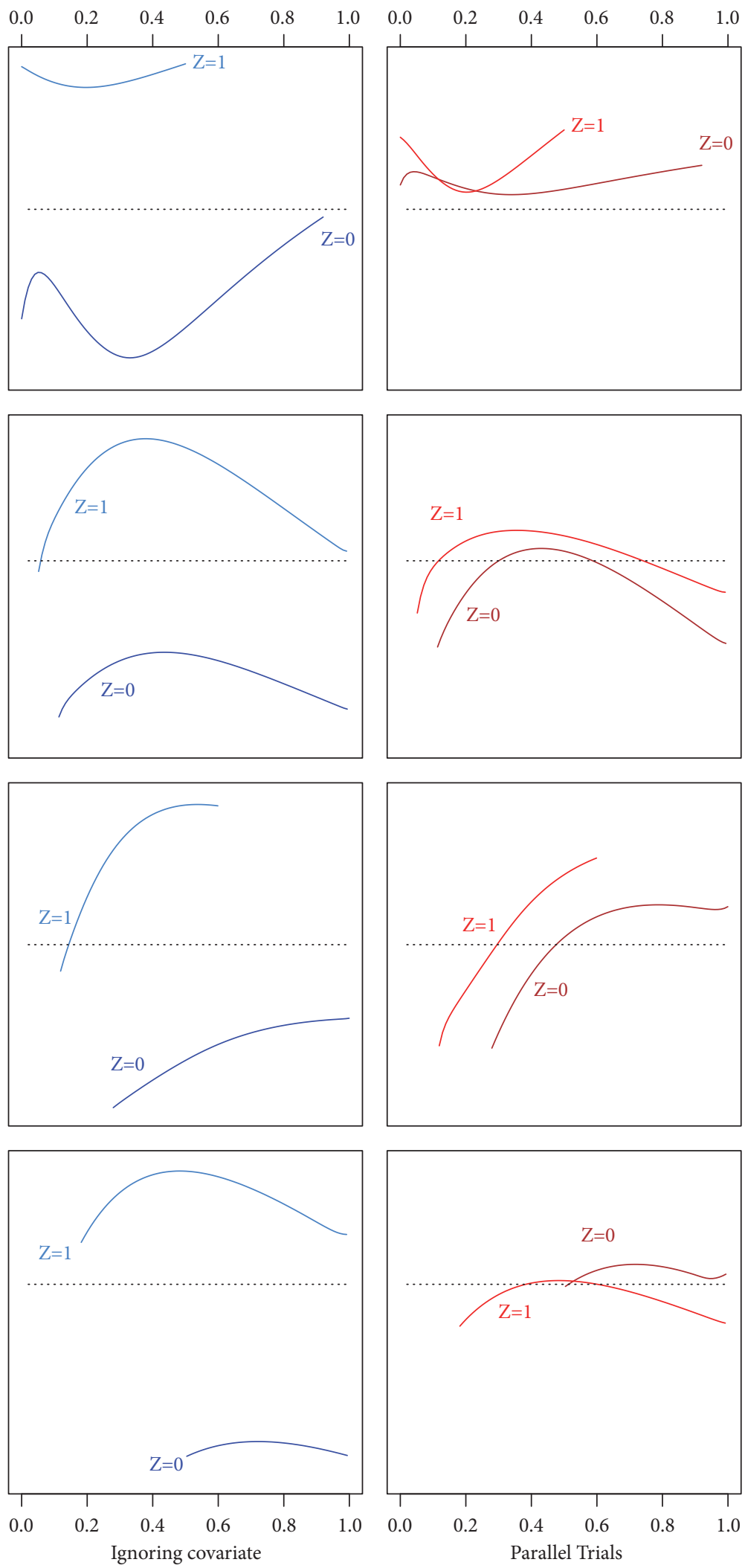

FIgURE 2: Pointwise average relative minimum distance from the true MTD curve to the estimated MTD curve with designs using a covariate (WC), ignoring the covariate (IC), and parallel trials (PT) under scenarios (a)-(d). 

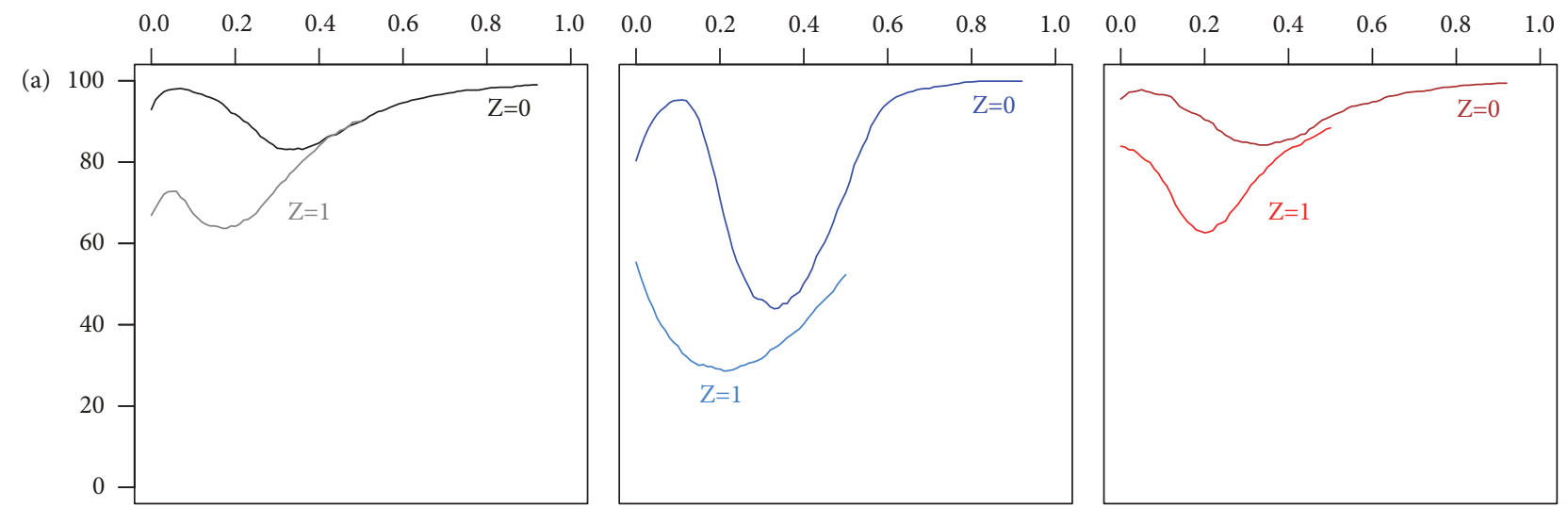

(b)
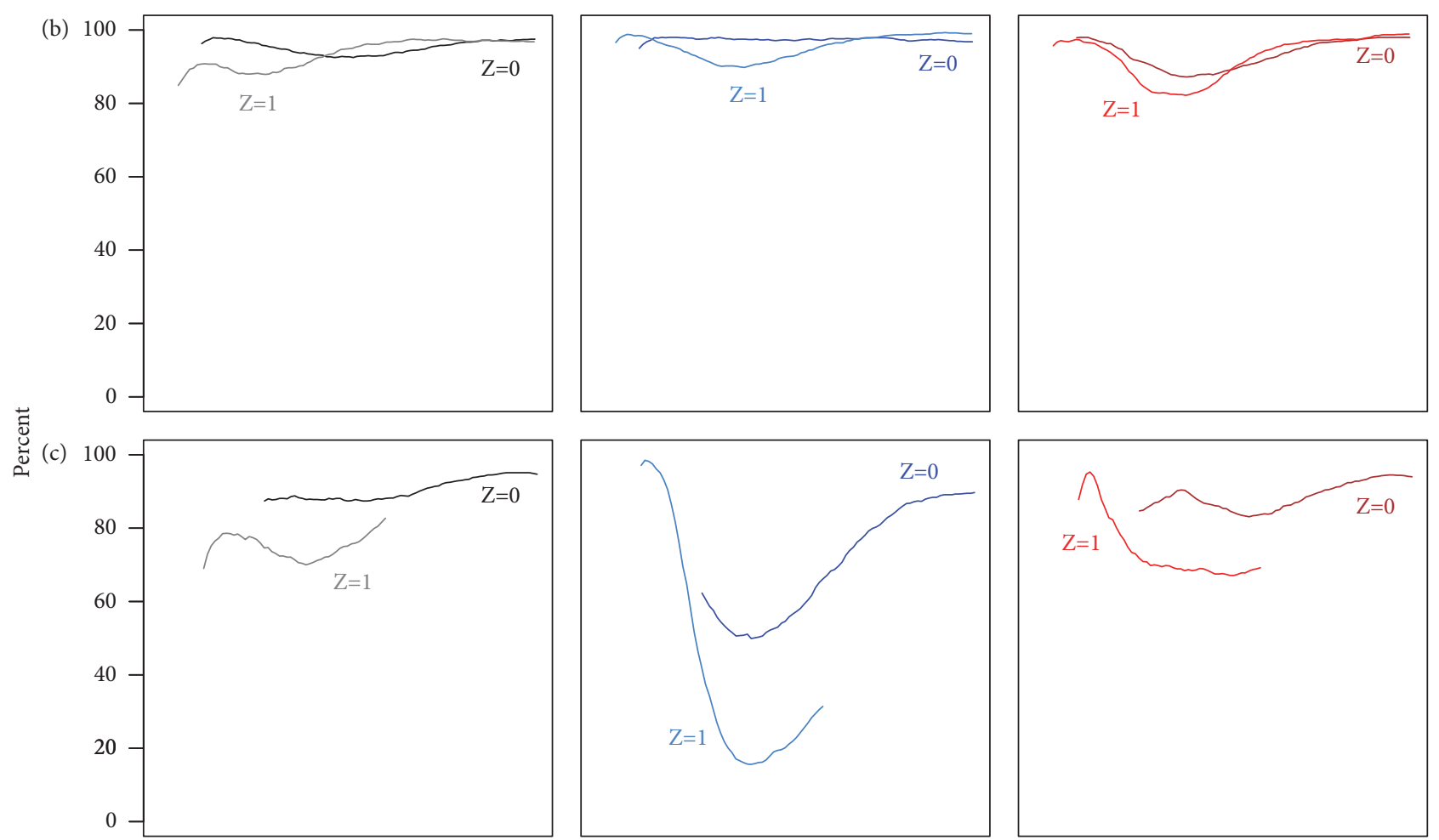

(d) 100
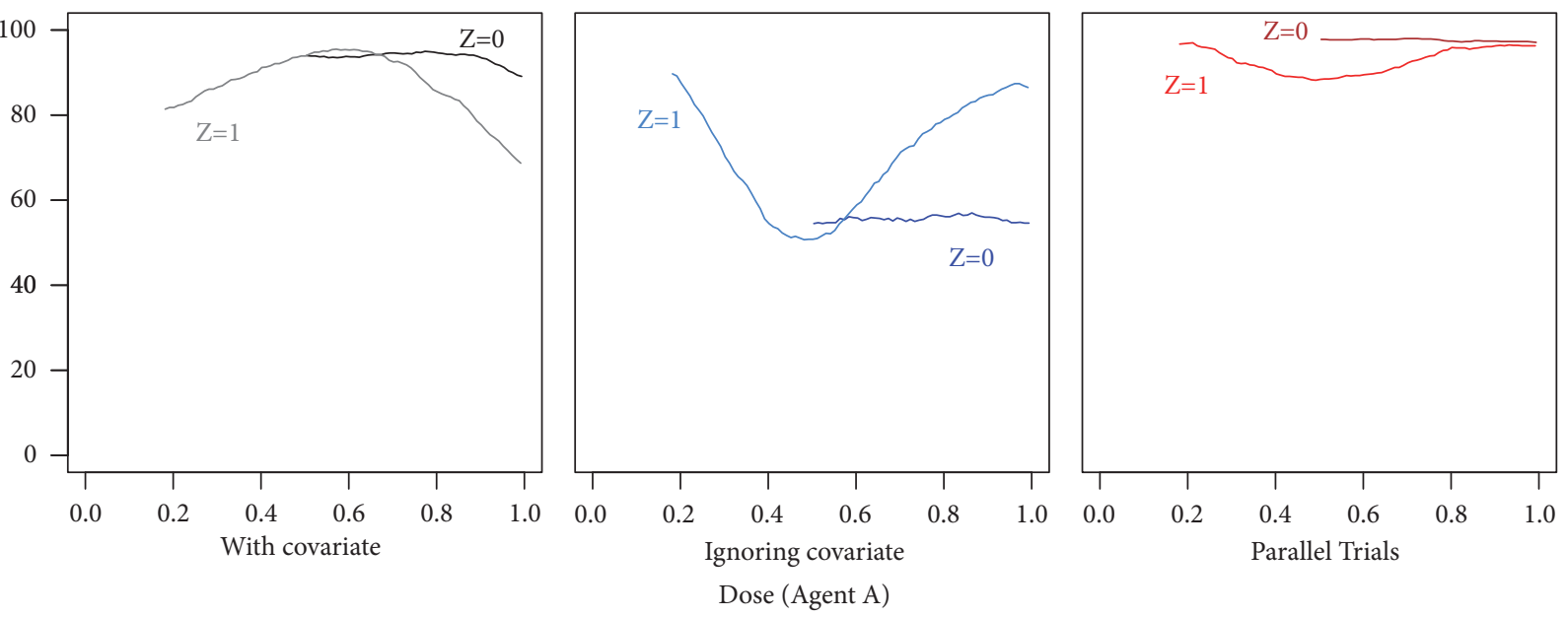

FIGURE 3: Pointwise percent of MTD recommendation for $p=0.2$ with designs using a covariate (WC), ignoring the covariate (IC), and parallel trials (PT) under scenarios (a)-(d). 
TABLE 2: A selected dose limiting toxicity scenario with $\theta=0.33$ for $Z=0,1$ considering discrete dose combinations. True MTDs are shown in bold.

\begin{tabular}{lcccccccccc}
\hline \multirow{2}{*}{ Dose level } & \multicolumn{1}{c}{$\mathrm{Z}=0$} \\
& 1 & 2 & 3 & 4 & 5 & 1 & 2 & 3 & 4 & 5 \\
\hline 5 & 0.25 & $\mathbf{0 . 3 3}$ & 0.40 & 0.48 & 0.70 & 0.45 & 0.53 & 0.60 & 0.68 & 0.90 \\
4 & 0.20 & 0.26 & $\mathbf{0 . 3 3}$ & 0.43 & 0.55 & 0.40 & 0.46 & 0.53 & 0.63 & 0.75 \\
3 & 0.13 & 0.16 & 0.24 & $\mathbf{0 . 3 3}$ & 0.39 & $\mathbf{0 . 3 3}$ & 0.36 & 0.44 & 0.53 & 0.59 \\
2 & 0.05 & 0.13 & 0.18 & 0.28 & $\mathbf{0 . 3 3}$ & 0.25 & $\mathbf{0 . 3 3}$ & 0.38 & 0.48 & 0.53 \\
1 & 0.001 & 0.05 & 0.13 & 0.20 & 0.27 & 0.201 & 0.25 & $\mathbf{0 . 3 3}$ & 0.40 & 0.47 \\
\hline
\end{tabular}

the MTD curve when $Z=0,1$. The other three misspecified models are shown in Figure S2.

\section{Discrete Dose Combinations}

In this section, we show how the proposed methodology can be applied to a prespecified discrete set of dose combinations.

4.1. Approach. Let $\left(x_{1}, \ldots, x_{r}\right)$ and $\left(y_{1}, \ldots, y_{s}\right)$ be the doses of agents $A$ and $B$, respectively. Following the notation of Section 2.1, $X_{\min , A}=x_{1}, Y_{\min , B}=y_{1}, X_{\max , A}=x_{r}, Y_{\max , B}=$ $y_{s}$, the doses are standardized to be in the interval $[0,1]$, and $z$ is a binary baseline covariate. Trial design proceeds using the algorithm described in Section 2.3 where the continuous doses recommended in steps (2) and (3) are rounded to the nearest discrete dose levels. At the end of the trial, a discrete set $\Gamma$ of dose combinations satisfying (i) and (ii) below is selected as MTDs. Let $C_{z, i}$ be the estimated MTD curve for $z=0,1$ at the end of the trial and denote by $d_{z}\left(\left(x_{j}, y_{k}\right), C_{z, i}\right)$ the Euclidean distance between the dose combination $\left(x_{j}, y_{k}\right)$ and $C_{z, i}$ for $z=0,1$ as in (12).

(i) Let $\Gamma_{z, A}=\bigcup_{t=1}^{r}\left\{\left(x_{t}, y\right): y=\right.$ $\left.\operatorname{argmin}_{y_{j}} d\left(\left(x_{t}, y_{j}\right), C_{z, i}\right)\right\}, \Gamma_{z, B}=\bigcup_{t=1}^{s}\left\{\left(x, y_{t}\right):\right.$ $\left.x=\operatorname{argmin}_{x_{j}} d\left(\left(x_{j}, y_{t}\right), C_{z, i}\right)\right\}$, and $\Gamma_{z, 0}=\Gamma_{z, A} \cap \Gamma_{z, B}$.

(ii) Let $\Gamma_{z}=\Gamma_{z, 0} \backslash\left\{\left(x^{*}, y^{*}\right): P\left(\left|P\left(D L T \mid\left(x^{*}, y^{*}\right), z\right)-\theta\right|>\right.\right.$ $\left.\left.\delta_{1} \mid D_{n}\right)>\delta_{2}\right\}$.

In (i), dose combinations closest to the MTD are selected by first minimizing the distances across the levels of drug A, then across the levels of drug B. In (ii), we exclude MTDs from (i) that either likely to be too toxic or too low. The design parameter $\delta_{1}$ is selected after consultation with a clinician and the parameter $\delta_{2}$ is selected after exploring a large number of scenarios for a given prospective trial. Following Tighiouart (2017) $[25], \delta_{1}=0.1, \delta_{2}=0.7$.

4.2. Operating Characteristics. The performance of the method is evaluated by calculating the percent of MTDs selection introduced in Tighiouart (2017) [25] estimating the probability that for a given scenario, a prospective trial will recommend a set of dose combinations that are all MTDs:

$$
\mathrm{PS}_{z}=100 \times \frac{1}{m} \sum_{i=1}^{m} \llbracket\left(\Gamma_{z, i} \subset \Gamma_{z, \delta}\right),
$$

for $z=0,1$, where $\Gamma_{z, \delta}=\left\{\left(x_{i}, y_{j}\right):\left|P\left(D L T \mid\left(x_{i}, y_{j}\right), z\right)-\theta\right|<\right.$ $\delta\}$ is the set of true MTDs such that the threshold parameter $\delta$ is fixed by a clinician. In the same way, the percent of selection at least $K$ dose combinations that are MTDs is

$$
\mathrm{PS}_{z}-\mathrm{K}=100 \times \frac{1}{m} \sum_{i=1}^{m} \llbracket\left(\left|\Gamma_{z, i} \cap \Gamma_{z, \delta}\right| \geq K\right),
$$

for $z=0,1$. In addition, the weighted average proportion of the recommended set of dose combinations which are MTDs discussed in [30] is given by

$$
\mathrm{S}_{\Gamma_{\delta}}=\frac{\sum_{i=1}^{m}\left|\Gamma_{z, i} \cap \Gamma_{z, \delta}\right|}{\sum_{i=1}^{m}\left|\Gamma_{z, i}\right|}
$$

for $z=0,1$.

4.3. Illustration. We present one scenario as shown in Table 2 with $r=s=5$ and the target probability of DLT is $\theta=0.33$. We simulated $m=1000$ trials using the sample size of $n=$ 40 patients with 20 patients per group, and the same vague priors for $\rho_{000}, \rho_{100}, \rho_{101}, \rho_{010}$ and $\eta$ from Section 3 to compare the three designs with a covariate, ignoring the covariate, and parallel trials.

Table 3 shows that the overall average DLT is $25.1 \%$ for the WC design and $24.5 \%$ for the IC design. In the group with $Z=0$, it is always far lower than $\theta$ and close to $\theta$ for the group with $Z=1$. The percent of trials with an excessive DLT rate is not noticeable for all designs where the highest values are observed when using the IC design.

Table 4 shows that the design using parallel trials has highest values for the percent of MTDs selection (PS), percent of selection of at least 3 dose combinations (S-3), 2 dose combinations (S-2), and 1 dose combination (S-1), and weighted average percent of the recommended set of dose combinations $\left(\mathrm{S}_{\Gamma_{\delta}}\right)$ statistics in the group with $Z=$ 0,1 . The IC design shows the lowest values for all operating characteristics in both groups; PT design presents smaller values than for WC design when $Z=0$, while shows higher values than for WC design when $Z=1$, except for $\mathrm{PS}_{z}-3$.

\section{Conclusion}

We described Bayesian adaptive designs for cancer phase I clinical trials using two drugs with continuous dose levels in the presence of a binary baseline covariate. The goal is to estimate the MTD curve in the two-dimensional Cartesian plane for a patient's specific baseline covariate value. The methodology extends the single agent trial design with a baseline covariate and two agents design without a covariate. In each case, vague priors were used to quantify the toxicity profile of each agent a priori. We used an algorithm for dose escalation where cohorts of two patients are enrolled simultaneously and the patients receive different dose combinations. We studied design operating characteristics of the method under four practical scenarios by comparing this method with the design that ignores the baseline covariate and design using parallel trials. In all simulations, we used a sample size of $n=40$ patients, 20 patients in each group. We 
TABLE 3: Operating characteristics summarizing trial safety for designs using a covariate (WC), ignoring the covariate (IC), and parallel trials (PT) considering discrete dose combinations.

\begin{tabular}{lccc}
\hline \multirow{2}{*}{ Design } & & Average $\%$ DLTs $(\%$ Trials: DLT rate $<\theta-0.1 ; \%$ Trials: DLT rate $>\theta+0.1)$ & $Z=1$ \\
\hline WC & Overall & Z $=0$ & $34.5(7.2 ; 17.4)$ \\
IC & $25.9(31.9 ; 2.0)$ & $17.3(78.5 ; 3.0)$ & $34.6(7.7 ; 17.5)$ \\
PT & $24.4(48.0 ; 0.0)$ & $14.2(85.6 ; 0.0)$ & $28.1(24.0 ; 5.0)$ \\
\hline
\end{tabular}

TABLE 4: Operating characteristics summarizing trial efficiency for $Z=0,1$ with designs using covariate (WC), ignoring covariate (IC), and parallel trials (PT) considering discrete dose combinations.

\begin{tabular}{lcccccc}
\hline Covariate & Design & PS & $\mathrm{PS}_{z}-3$ & $\mathrm{PS}_{z}-2$ & $\mathrm{PS}_{z}-1$ & $\mathrm{~S}_{\mathrm{\Gamma}_{\delta}}$ \\
\hline \multirow{3}{*}{$\mathrm{Z}=0$} & WC & 47.3 & 35.3 & 51.3 & 80.1 & 66.3 \\
& IC & 19.6 & 14.9 & 23.0 & 51.6 & 37.9 \\
& PT & 46.2 & 9.9 & 33.6 & 58.3 & 56.4 \\
\hline \multirow{3}{*}{$\mathrm{Z}=1$} & WC & 55.3 & 25.7 & 72.5 & 85.5 & 71.6 \\
& IC & 47.6 & 17.9 & 59.5 & 79.6 & 62.7 \\
& PT & 81.6 & 16.2 & 71.4 & 88.0 & 88.9 \\
\hline
\end{tabular}

found that in general, the methodology is safe in terms of the probability that a prospective trial will result in an excessively high number of DLTs when accounting for a significant covariate. We used several measures to assess the efficiency of the estimate of the MTD. In the presence of a practically significant baseline covariate, the design with a covariate had a smaller pointwise average bias and a higher percent of MTD recommendation relative to a design which ignores the covariate and similar performance to parallel trials when the groups were balanced. When the two true MTD curves are very close, including a baseline covariate in the model results in a slightly higher but still negligible bias and a small reduction in percent of MTD recommendation relative to the design that ignores this covariate. Therefore, we stand to lose little if we include a practically not important covariate in the model. We further showed how this methodology is adapted to the discrete dose combinations and proposed statistics estimating the probability that a prospective trial will recommend a set of dose combinations that are all MTDs for a given scenario. The statistics are used in evaluating the performance of the proposed design with a covariate as compared to other designs ignoring the covariate and using parallel trials.

\section{Data Availability}

The data used to support the findings of this study are available from the corresponding author upon request.

\section{Disclosure}

An earlier version from the manuscript has been presented as poster in the Joint Statistical Meeting in Chicago, 2016 [31].

\section{Conflicts of Interest}

The authors declare that they have no conflicts of interest.

\section{Acknowledgments}

This research was partially funded by grants from the National Institute of Health, Grant Number 1R01CA18848001A1 (Mourad Tighiouart, Sungjin Kim), and the National Center for Research Resources, Grant UL1RR033176, and is now at the National Center for Advancing Translational Sciences, Grant UL1TR000124 (Mourad Tighiouart), and P01 CA098912 (Mourad Tighiouart, Márcio Augusto Diniz).

\section{Supplementary Materials}

(i) Table S1: operating characteristics summarizing trial safety for designs using a covariate (WC), ignoring the covariate (IC), and parallel trials (PT) considering continuous dose combinations, $\theta=0.33$. (ii) Figure S1: pointwise average relative minimum distance from the true MTD curve to the estimated MTD curve with designs using a covariate (WC), ignoring the covariate (IC), and parallel trials (PT) under scenarios (a)-(d). (iii) Figure S2: pointwise percent of MTD recommendation for $p=0.2$ with designs using a covariate (WC), ignoring the covariate (IC), and parallel trials (PT) under scenarios (a)-(d). (Supplementary Materials)

\section{References}

[1] J. S. Lopez and U. Banerji, "Combine and conquer: Challenges for targeted therapy combinations in early phase trials," Nature Reviews Clinical Oncology, vol. 14, no. 1, pp. 57-66, 2017.

[2] P. F. Thall, R. E. Millikan, P. Mueller, and S.-J. Lee, "Dose-finding with two agents in Phase I oncology trials," Biometrics: Journal of the International Biometric Society, vol. 59, no. 3, pp. 487-496, 2003.

[3] K. Wang and A. Ivanova, "Two-dimensional dose finding in discrete dose space," Biometrics: Journal of the International Biometric Society, vol. 61, no. 1, pp. 217-222, 2005.

[4] G. Yin and Y. Yuan, "A latent contingency table approach to dose finding for combinations of two agents," Biometrics: Journal of the International Biometric Society, vol. 65, no. 3, pp. 866-875, 2009.

[5] G. Yin and Y. Yuan, "Bayesian dose finding in oncology for drug combinations by copula regression," Journal of the Royal Statistical Society: Series C (Applied Statistics), vol. 58, no. 2, pp. 211-224, 2009.

[6] T. M. Braun and S. Wang, "A hierarchical Bayesian design for phase 1 trials of novel combinations of cancer therapeutic 
agents," Biometrics: Journal of the International Biometric Society, vol. 66, no. 3, pp. 805-812, 2010.

[7] N. A. Wages, M. R. Conaway, and J. O’Quigley, "Continual reassessment method for partial ordering," Biometrics: Journal of the International Biometric Society, vol. 67, no. 4, pp. 1555$1563,2011$.

[8] N. A. Wages, M. R. Conaway, and J. O'Quigley, "Dose-finding design for multi-drug combinations," Clinical Trials, vol. 8, no. 4, pp. 380-389, 2011.

[9] M. J. Sweeting and A. P. Mander, "Escalation strategies for combination therapy Phase i trials," Pharmaceutical Statistics, vol. 11, no. 3, pp. 258-266, 2012.

[10] Y. Shi and G. Yin, "Escalation with overdose control for phase I drug-combination trials," Statistics in Medicine, vol. 32, no. 25, pp. 4400-4412, 2013.

[11] M. Tighiouart, S. Piantadosi, and A. Rogatko, "Dose finding with drug combinations in cancer phase I clinical trials using conditional escalation with overdose control," Statistics in Medicine, vol. 33, no. 22, pp. 3815-3829, 2014.

[12] M.-K. Riviere, Y. Yuan, F. Dubois, and S. Zohar, "A Bayesian dose-finding design for drug combination clinical trials based on the logistic model," Pharmaceutical Statistics, vol. 13, no. 4, pp. 247-257, 2014.

[13] A. P. Mander and M. J. Sweeting, "A product of independent beta probabilities dose escalation design for dual-agent phase I trials," Statistics in Medicine, vol. 34, no. 8, pp. 1261-1276, 2015.

[14] R. K. Ramanathan, M. J. Egorin, C. H. M. Takimoto et al., "Phase I and pharmacokinetic study of imatinib mesylate in patients with advanced malignancies and varying degrees of liver dysfunction: A study by the national cancer institute organ dysfunction working group," Journal of Clinical Oncology, vol. 26, no. 4, pp. 563-569, 2008.

[15] T. B. Leal, S. C. Remick, C. H. Takimoto et al., "Dose-escalating and pharmacological study of bortezomib in adult cancer patients with impaired renal function: A National Cancer Institute Organ Dysfunction Working Group Study," Cancer Chemotherapy and Pharmacology, vol. 68, no. 6, pp. 1439-1447, 2011.

[16] T. Satoh, T. Ura, Y. Yamada et al., "Genotype-directed, dosefinding study of irinotecan in cancer patients with UGT1A1 ${ }^{\star} 28$ and/or UGT1A1 ${ }^{\star} 6$ polymorphisms," Cancer Science, vol. 102, no. 10, pp. 1868-1873, 2011.

[17] P. M. LoRusso, K. Venkatakrishnan, R. K. Ramanathan et al., "Pharmacokinetics and safety of bortezomib in patients with advanced malignancies and varying degrees of liver dysfunction: Phase I NCI Organ Dysfunction Working Group Study NCI-6432," Clinical Cancer Research, vol. 18, no. 10, pp. 29542963, 2012.

[18] K.-P. Kim, H.-S. Kim, S. J. Sym et al., "A UGT1A1²8 and ${ }^{\star} 6$ genotype-directed phase $\mathrm{i}$ dose-escalation trial of irinotecan with fixed-dose capecitabine in Korean patients with metastatic colorectal cancer," Cancer Chemotherapy and Pharmacology, vol. 71, no. 6, pp. 1609-1617, 2013.

[19] J. O'Quigley, L. Z. Shen, and A. Gamst, “Two-sample continual reassessment method," Journal of Biopharmaceutical Statistics, vol. 9, no. 1, pp. 17-44, 1999.

[20] M. Tighiouart, G. Cook-Wiens, and A. Rogatko, "Escalation with overdose control using ordinal toxicity grades for cancer phase I clinical trials," Journal of Probability and Statistics, Art. ID 317634, 17 pages, 2012.
[21] J. O'Quigley and X. Paoletti, “Continual reassessment method for ordered groups," Biometrics: Journal of the International Biometric Society, vol. 59, no. 2, pp. 430-440, 2003.

[22] A. Ivanova and K. Wang, "Bivariate isotonic design for dosefinding with ordered groups," Statistics in Medicine, vol. 25, no. 12, pp. 2018-2026, 2006.

[23] Z. Yuan and R. Chappellb, "Isotonic designs for phase I cancer clinical trials with multiple risk groups," Clinical Trials, vol. 1, no. 6, pp. 499-508, 2004.

[24] M. R. Conaway, "A design for phase I trials in completely or partially ordered groups," Statistics in Medicine, vol. 36, no. 15, pp. 2323-2332, 2017.

[25] M. Tighiouart, Q. Li, and A. Rogatko, "A Bayesian adaptive design for estimating the maximum tolerated dose curve using drug combinations in cancer phase I clinical trials," Statistics in Medicine, vol. 36, no. 2, pp. 280-290, 2017.

[26] J. Babb, A. Rogatko, and S. Zacks, "Cancer phase I clinical trials: Efficient dose escalation with overdose control," Statistics in Medicine, vol. 17, no. 10, pp. 1103-1120, 1998.

[27] M. Tighiouart, Q. Li, S. Piantadosi, and A. Rogatko, "A Bayesian Adaptive Design for Combination of Three Drugs in Cancer Phase I Clinical Trials," American Journal of Biostatistics, vol. 6, no. 1, pp. 1-11, 2016.

[28] M. Plummer, "JAGS: A program for analysis of Bayesian graphical models using Gibbs sampling," in Proceedings of the 3 rd international workshop on distributed statistical computing, vol. 124, p. 125, Vienna, 2003.

[29] M. Tighiouart and A. Rogatko, "Dose finding with escalation with overdose control (EWOC) in cancer clinical trials," Statistical Science. A Review Journal of the Institute of Mathematical Statistics, vol. 25, no. 2, pp. 217-226, 2010.

[30] M. A. Diniz, Q. Li, and M. Tighiouart, "Dose Finding for Drug Combination in Early Cancer Phase I Trials Using Conditional Continual Reassessment Method," Journal of Biometrics Biostatistics, vol. 8, 2017.

[31] S. Kim, M. A. Diniz, and M. Tighiouart, "A Bayesian Adaptive," in Proceedings of the Design in Cancer Phase I Trials using Dose Combinations in the Presence of a Baseline Covariate, A. S. Association, Ed., pp. 1336-1347, Alexandria, VA, 2016. 


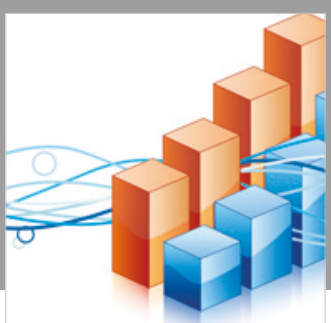

Advances in

Operations Research

\section{-n-m}
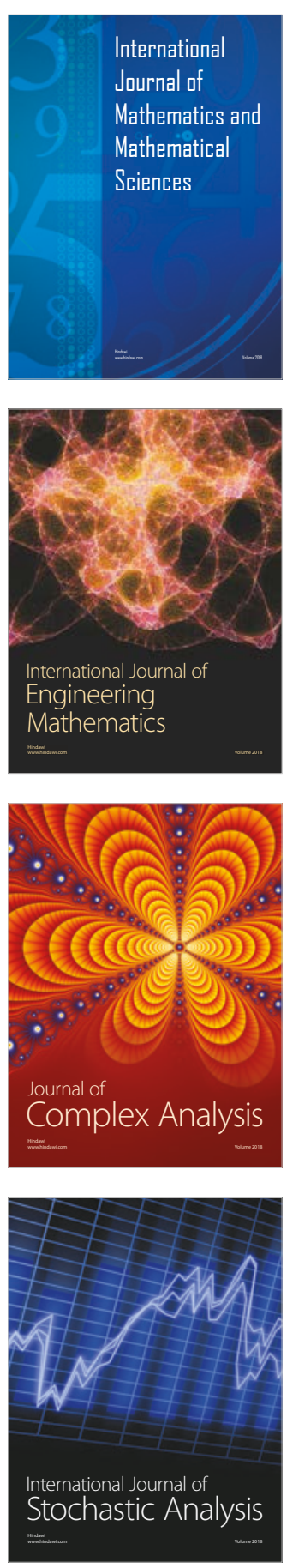
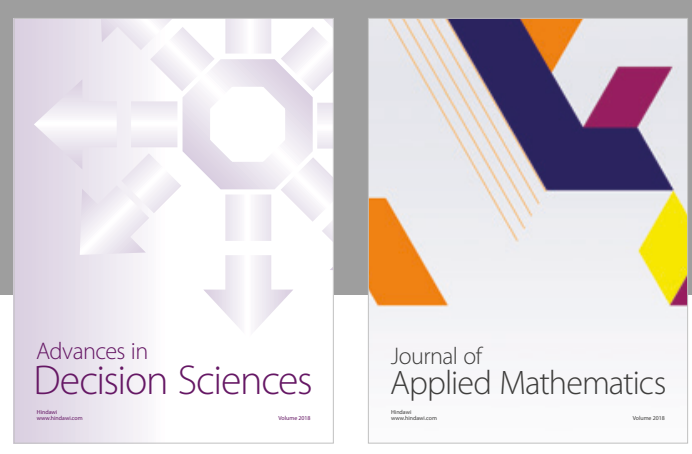

Journal of

Applied Mathematics
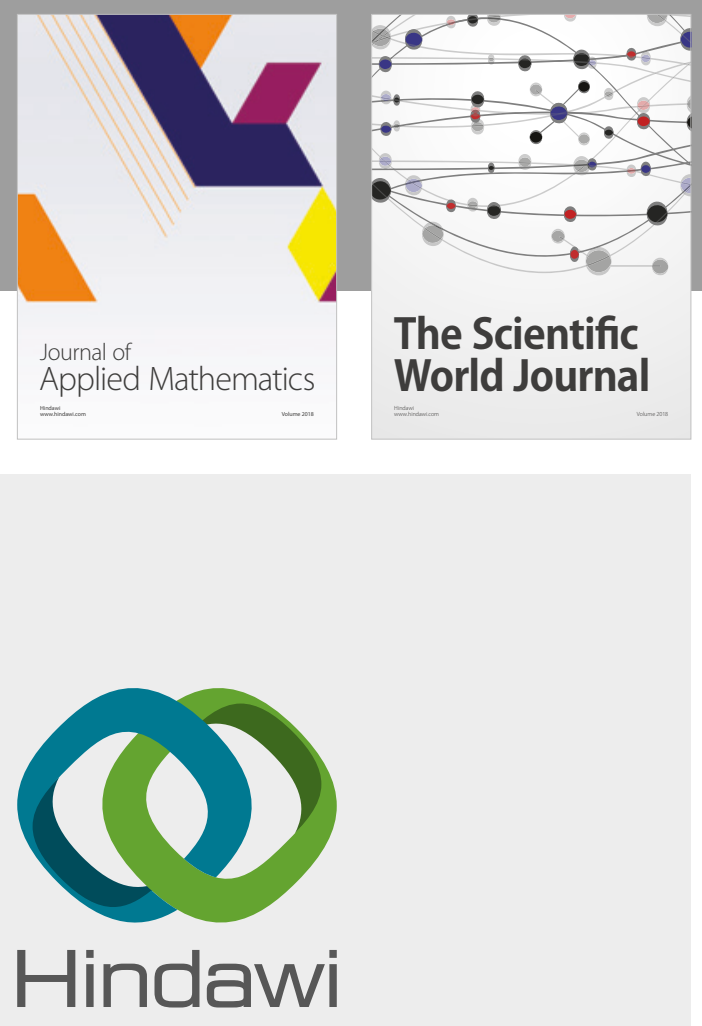

Submit your manuscripts at

www.hindawi.com

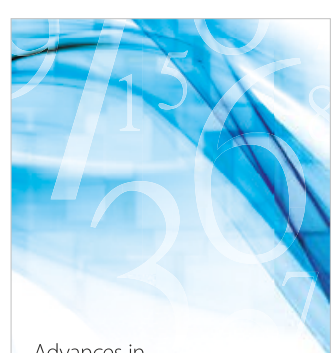

Advances in
Numerical Analysis
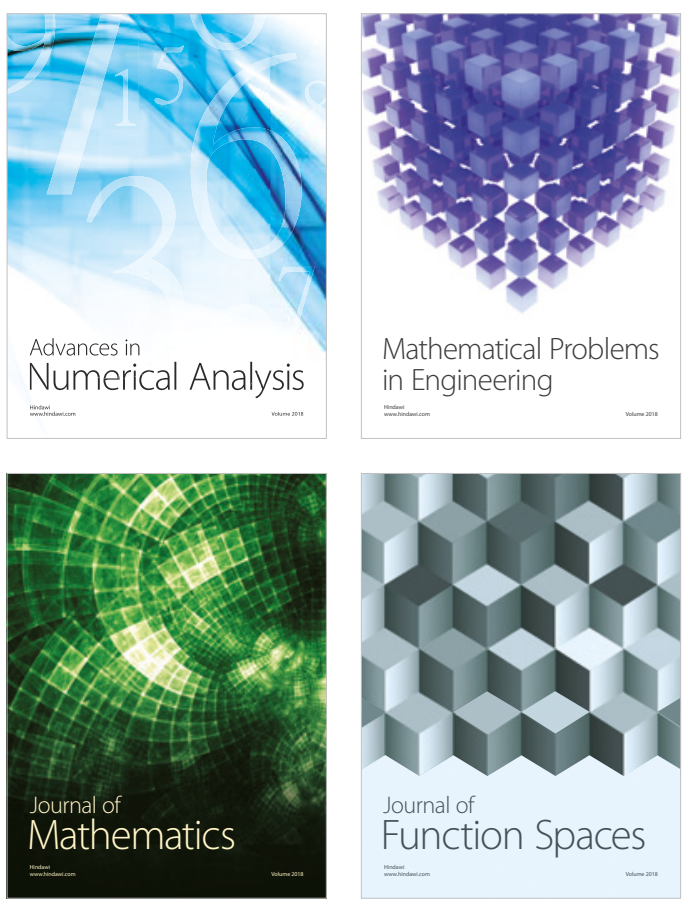

Mathematical Problems in Engineering

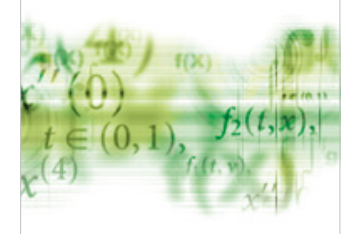

International Journal of

Differential Equations

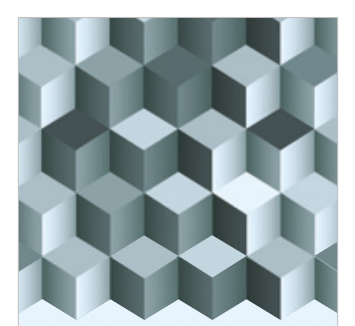

Journal of

Function Spaces

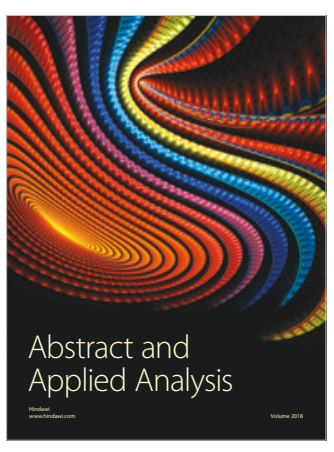

The Scientific

World Journal

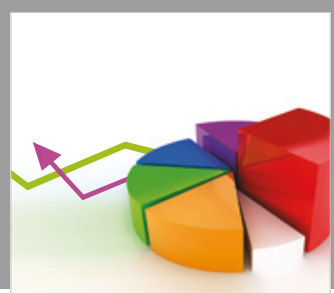

Journal of

Probability and Statistics
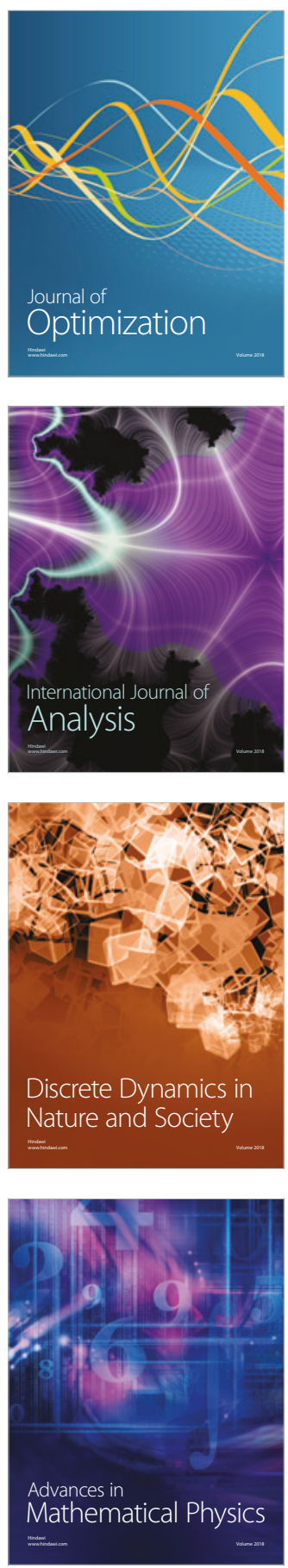